\title{
Matrix metalloproteinase 10 is associated with survival in patients with esophageal squamous cell carcinoma
}

Esophageal squamous cell carcinoma (ESCC) is an aggressive malignancy with a poor prognosis. A Chinese study has identified overexpression of matrix metalloproteinase 10 (MMP10) in samples of ESCC tumors. As this overexpression seems to be linked to survival, MMP10 could be used as a prognostic marker.

The authors of the study used tissue microarray and immunohistochemistry

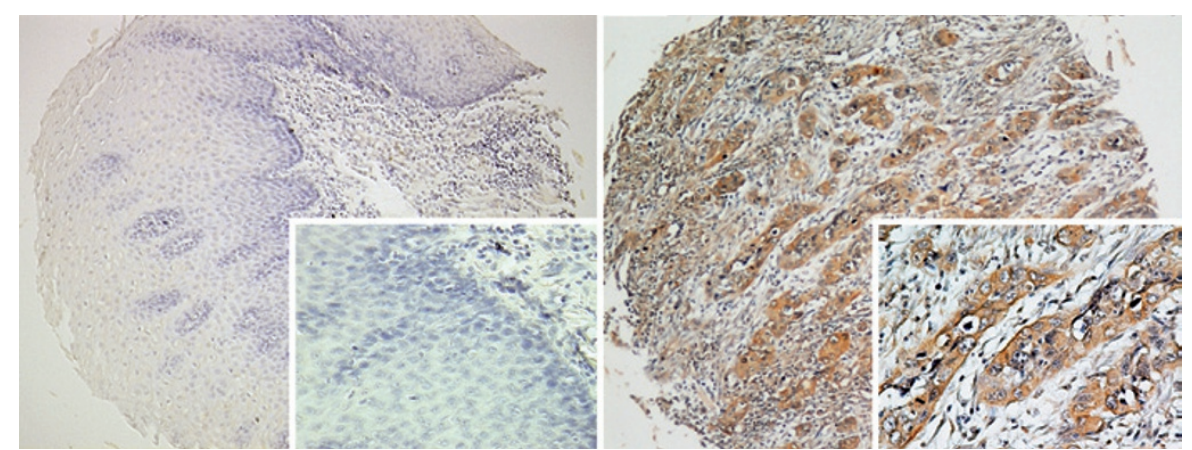

An immunohistochemistry staining of matrix metalloproteinase 10 in esophageal squamous cell carcinoma tumor tissue (right) and its normal (left) counterpart from tissue microarray. Courtesy of L. Fu.

staining to analyze 300 pairs of ESCC tissue specimens and nontumorous tissue samples collected from patients in a region of China with a high risk of ESCC.

Overexpression of MMP10 was detected at the mRNA level (65\%) and the protein level (79\%) in the ESCC tumors, but not in nontumor esophageal tissue. Although overall MMP10 overexpression was not associated with survival, patients with early stage ESCC and overexpression of MMP10 had poorer survival than those who did not have overexpression at this stage. Therefore, "overexpression of MMP10 ... could be used as a prognostic marker for Chinese ESCC patients with early clinical stages of the disease," explain authors Li Fu and Xin-Yuan Guan.

The researchers plan to study the functional role of MMP10 and the molecular mechanisms of the development and progression of ESCC. They also hope to "examine the expression levels of MMP10 in serum samples from patients with ESCC and figure out its clinical significance".

\section{Claire Greenhill}

Original article Liu, H. et al. Overexpression of matrix metalloproteinase 10 is associated with poor survival in patients with early stage of esophageal squamous cell carcinoma. Dis. Esophagus doi:10.1111/j.1442 2050.2011.01284.x 